\title{
Looking back at 2021
}

\author{
As 2021 comes to an end, we take this opportunity to look back through the pages of Nature Structural \& Molecular \\ Biology and consider some of the year's highlights.
}

2 021 has been a year of continued disruption, but also of glimmers of hope and renewed confidence in the power of science to provide answers and help address some of the societal challenges we face. Here we present an overview of a few of the most significant advances we have published in the last year. As the global COVID-19 pandemic has continued to dominate our lives, it is not surprising that the development of antiviral treatments against COVID-19 has been of great interest. With the clinical trials of the oral antiviral drug molnupirovir, now approved in the United Kingdom for COVID-19 treatment, we were excited to publish a structural and biochemical investigation of the 'Mechanism of molnupiravir-induced SARS-CoV-2 mutagenesis' by the Cramer lab, accompanied by a News \& Views piece presenting an overview of how molnupirovir "disrupts the fidelity of SARS-CoV-2 genome replication and prevents viral propagation by fostering error accumulation in a process referred to as 'error catastrophe"', by Brandon Malone and Elizabeth A. Campbell. Identification of other antiviral molecules that may offer efficient treatment for COVID-19 was provided by Xu et al., who found that suramin, a 100-year-old anti-parasitic drug, is also an inhibitor of the SARS-CoV-2 RNA-dependent RNA polymerase, and work from Xiao et al. showing that engineered ACE2 molecules may function as a potential anti-SARS-CoV-2 agents. Another possible therapeutic approach targets the SARS-CoV-2 pseudoknot, as illustrated in a study by Zhang et al, wherein the cryo-EM structure of the pseudoknot-forming SARS-CoV-2 frameshift-stimulation element was used to guide the design of antisense oligonucleotides that impair viral replication, while work by Sauer et al. presented a 'Structural basis for broad coronavirus neutralization'. Together, these studies highlight how the powerful tools of molecular and structural biology uncover mechanisms of action that inform COVID-19 therapies. As a journal, we are also interested in the wider perspective of the scientists leading these projects. In the beginning of the year, we were delighted to feature some of the researchers advancing 'Structural biology in the fight against COVID-19', and we look forward to continuing to work with the community to support these efforts.

Another scientific highlight of the year for us was the announcement that the 2021 Nobel Prize in Physiology or Medicine had been awarded to David Julius and Ardem Patapoutian "for their discoveries of receptors for temperature and touch." We were thrilled to help put together a Nature Portfolio Collection featuring publications from the winners, some essential reviews on the topic, and further exciting research on various aspects of TRP and PIEZO channels and their roles in physiology and disease. The collection included two studies we published from the Lee and Sobolevsky labs, which presented cryo-EM structures of the sensory ion channels TRPV1 and TRPV3 at different temperatures, revealing the mechanistic principles of heat-dependent gating. The clinical relevance of the research into TRP and PIEZO channels is underscored by the fact that in addition to sensing temperature and touch, respectively, they also have a role in pain sensation. Understanding and treating chronic pain is an essential front in the battle against the worldwide opioid crisis. Structural and molecular studies of pain receptors can result in promising alternatives to opioid analgesics, as illustrated by the recent study published in Nature wherein Draper-Joyce et al. show that a positive allosteric modulator of the adenosine A1 receptor (A1R) yields analgesia by stabilizing the agonist-A1R-G protein complex. The importance of this study was discussed in a News \& Views piece by Grégory Scherrer and colleagues, which highlighted the potential of this modulator for non-opioid treatment of chronic pain.

2021 also marked the 50th anniversary of the Protein Data Bank (PDB)! To celebrate the PDB, which was first announced on 20 October 1971 in Nature New Biology, we and our colleagues at Nature Methods presented a collection of articles reflecting on the past and future of structural biology. A few short months later, the future arrived with the publication of AlphaFold2, an artificial-intelligence method for protein-structure prediction. With the full potential of AlphaFold2 still to be unlocked, our authors have corresponded with us and our readers to discuss the potentials and current limits of AlphaFold 2 and what the future might hold. We have been following this conversation with great interest and look forward to presenting further insights of our authors in NSMB.

AlphaFold2 also provides an example of the power of open data. Without the efforts of not only the structural biology community to archive its structures in the PDB, but also the wider scientific community that has shared protein sequence information and genome sequencing data in public repositories located all over the world, there would be no data for AlphaFold 2 to build upon and generate its predictions. This triumph highlights the power of science when it follows the principles of transparency, openness, collegiality and cooperation. Looking forward, we strive to continue to help uphold these principles and support our community of readers and authors.

Published online: 6 December 2021 https://doi.org/10.1038/s41594-021-00704-4 